\title{
A Parallel-Strip Balun for Wideband Frequency Doubler
}

\author{
Leung Chiu and Quan Xue \\ Department of Electronic Engineering, City University of Hong Kong, Hong Kong \\ Correspondence should be addressed to Leung Chiu; eechiuleung@yahoo.com.hk \\ Received 18 September 2013; Revised 2 November 2013; Accepted 9 November 2013 \\ Academic Editor: Yong Xin Guo
}

Copyright (C) 2013 L. Chiu and Q. Xue. This is an open access article distributed under the Creative Commons Attribution License, which permits unrestricted use, distribution, and reproduction in any medium, provided the original work is properly cited.

\begin{abstract}
A parallel-strip phase inverter with a pair of simple impedance matching networks is designed. The phase inverter introduces the almost frequency independent $180^{\circ}$ phase shift and was employed in the wideband parallel-strip balun. The balun was designed and measured with the maximum magnitude imbalance of $0.5 \mathrm{~dB}$ and the maximum phase imbalance of $6.0^{\circ}$. The proposed balun is used as input network for the wideband balanced frequency doubler. The proposed frequency doubler achieves significant conversion gain from $0.1 \mathrm{GHz}$ to $1.7 \mathrm{GHz}$. The frequency doubler achieves $7.4 \mathrm{~dB}$ conversion gain and $23 \mathrm{~dB}$ fundamental signal suppression at $1 \mathrm{GHz}$.
\end{abstract}

\section{Introduction}

Differential radio frequency (RF) circuits are commonly found in the integrated circuits for wireless communication systems. The RF ports of these chips are not standalone, but they consist of both "positive" and "negative" terminals. However, most of the off-chip RF components such as antenna and switch are single-ended [1]. Balun, that, is a device for conversion between a single-ended signal and differential signals, is essential for the entire wireless module [2]. The differential signals are composed of two separated signals equal in magnitude but $180^{\circ}$ out-of-phase. Differential amplifiers and differential oscillators outperform single-ended circuits in terms of the even-order harmonic signal and commonmode noise suppressions [3]. A high performance balun design is critical for integrating the single-end and differential circuit in an efficient way. Besides, impedance bandwidth, phase imbalance, and magnitude imbalance are the three important design issues for the balun design.

Wilkinson power divider divides signal with high portto-port isolation, and the impedance of all ports is matched [4]. However, the divided signals are in-phase. Additional and fixed $180^{\circ}$ phase shifter is required for the balun based on the Wilkinson power divider. The frequency responses of both insertion loss and phase flatness of the phase shifter are keys of the balun performance. Many high performance balun designs based on the Wilkinson power divider were reported in $[1,4]$. In [1], the $180^{\circ}$ phase shifter was based on the combination of open circuit and short circuit stubs and microstrip lines. The measured impedance bandwidth is about $64 \%$. Another work based on microstrip metamaterial lines that was designed using lumped elements achieved impedance bandwidth of about 77\% [4].

A parallel-strip line is a balanced transmission line, which consists of two conductors separated by a dielectric substrate. The parallel-strip line achieves a wide range of characteristic impedance lines and high performance balanced microwave components [5]. The parallel-strip phase inverter is realized by interconnecting the upper and lower conductors of a $\mathrm{PCB}$ through the two metal vias. This phase inverter introduces a very wideband $180^{\circ}$ phase shift. The parallel-strip inverter was successfully applied to filter and directional coupler designs with performance enhancement $[6,7]$.

In the balanced frequency doubler design, the two identical nonlinear devices such as biased transistors and diodes were fed by a differential signal that is generated from the balun by single signal source. The output signals from the two devices are combined by in-phase power combiner. Meanwhile, the even-order harmonic signals are combined and extracted at the output port, and the oddorder harmonics signals are suppressed $[8,9]$. However, the bandwidth of a frequency doubler is limited by the balun. In this paper, a wideband balun based on Wilkinson power divider and parallel-strip inverter is proposed. Besides, a 


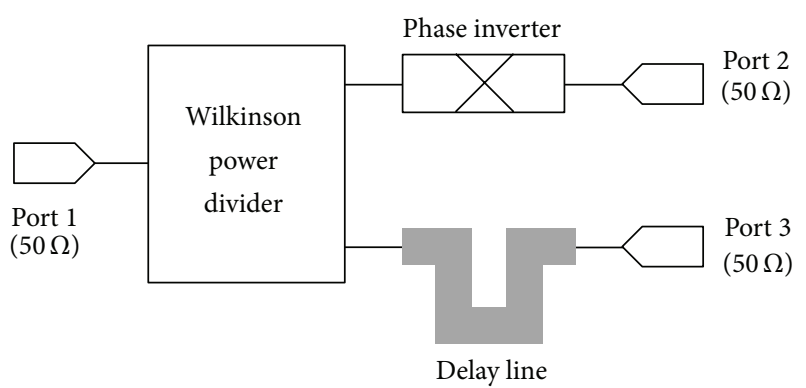

(a)

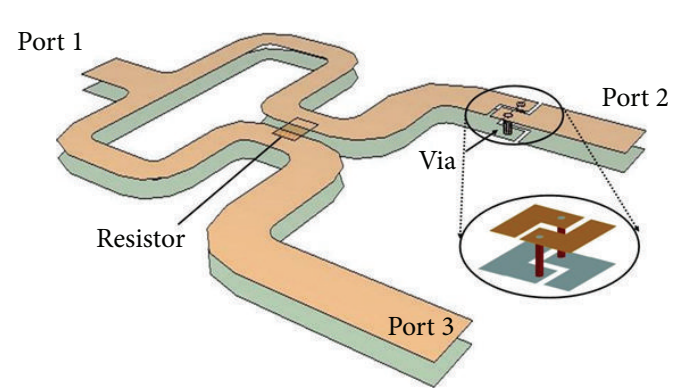

(b)

Figure 1: (a) Circuit diagram of the parallel-strip balun. (b) 3D view of the parallel-strip balun.

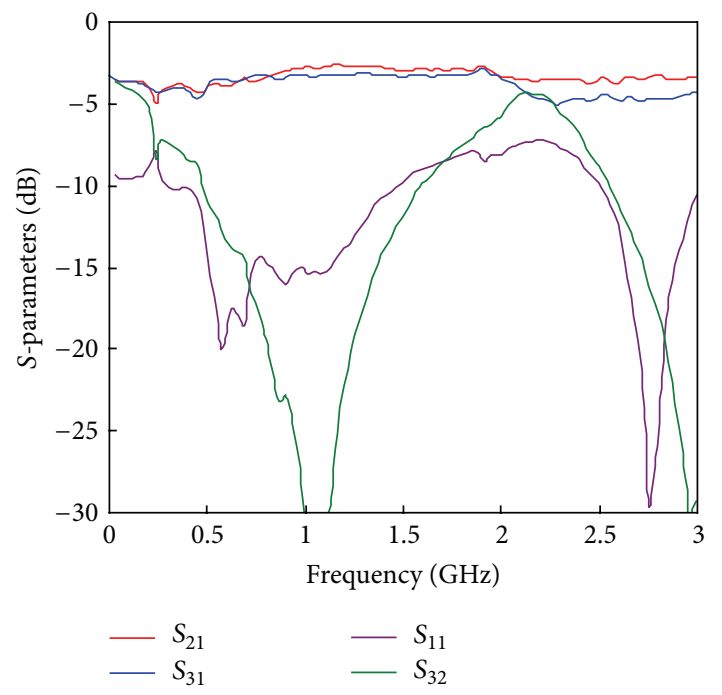

(a)

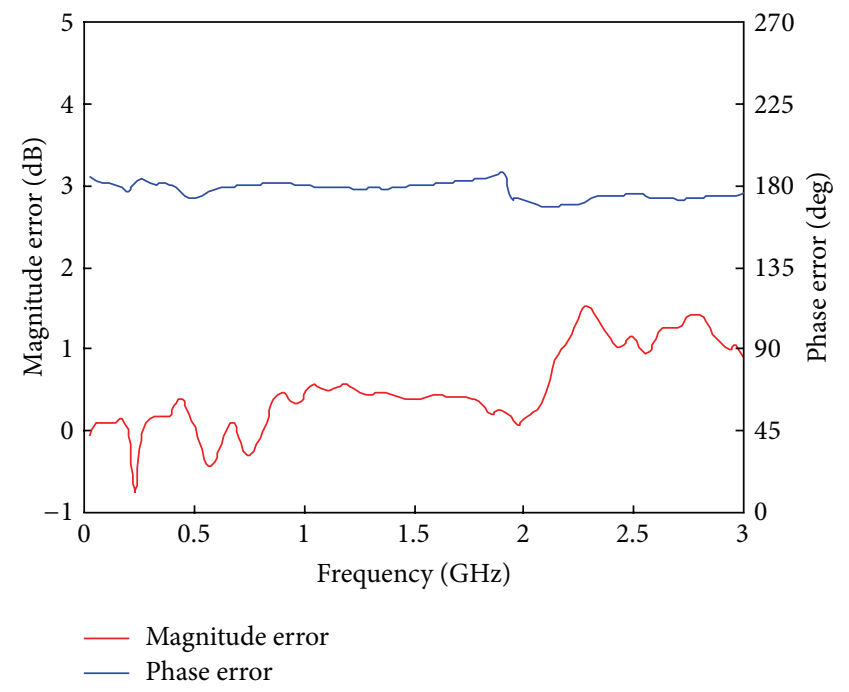

(b)

FIGURE 2: (a) Measured frequency responses of the magnitudes of the $S$-parameters of the balun. (b) Measured magnitude error and phase error of the balun.

wideband frequency doubler based on the proposed balun is presented.

\section{Parallel-Strip Balun}

The proposed balun consists of three parts, a parallel-strip Wilkinson power divider, a parallel-strip phase inverter, and a section of delay parallel-strip line. The circuit diagram and the geometry of the balun are shown in Figure 1. The proposed balun is designed at the centre frequency of $1.0 \mathrm{GHz}$ and it is simulated by the full-wave electromagnetic simulation software, Zeland IE3D [10]. The parallel-strip phase inverter is the core of the proposed balun and it introduces an approximately $180^{\circ}$ to the one of the divided signal. The design and the application of the parallel-strip inverter are reported in $[6,7]$. There are two lumped $50 \Omega$ isolation resistors mounted on both the upper and lower layers [5]. Figure 2 shows its measured frequency responses of the magnitudes of the four $S$-parameters. The measured bandwidth with full considerations of impedance matching $\left|S_{11}\right|,\left|S_{22}\right|$, and $\left|S_{33}\right| \leq-10 \mathrm{~dB}$ and isolation bandwidth $\left|S_{32}\right| \leq-10 \mathrm{~dB}$ is about $100 \%(0.48 \mathrm{GHz}-1.44 \mathrm{GHz})$. Within the above frequency range, the maximum magnitude error $\left|S_{12} / S_{13}\right|(\mathrm{dB})$ and the maximum phase error $\left|\angle S_{12}-\angle S_{13}-180^{\circ}\right|$ are $0.50 \mathrm{~dB}$ and $6.0^{\circ}$, respectively.

\section{Proposed Frequency Doubler}

Figure 3 shows the structure and the photograph of the balanced frequency doubler. It consists of two identical nonlinear devices, one input balun and one output Wilkinson power combiner. The biased bipolar junction transistor in common emitter configuration is closed for the nonlinear device since it achieves conversion gain. Diode can be used, but conversion loss is achieved instead of conversion gain. The conversion gain is maximized if Class- $\mathrm{B}$ biasing condition of the two transistors is chosen. The two nonlinear devices are governed by

$$
\begin{aligned}
& y_{1}=a_{1} x_{1}+a_{2} x_{1}^{2}+a_{3} x_{1}^{3}+a_{4} x_{1}^{4}+a_{5} x_{1}^{5}+\cdots, \\
& y_{2}=a_{1} x_{2}+a_{2} x_{2}^{2}+a_{3} x_{2}^{3}+a_{4} x_{2}^{4}+a_{5} x_{2}^{5}+\cdots,
\end{aligned}
$$

where $x_{1}$ and $x_{2}$ are the inputs of the two nonlinear devices and $y_{1}$ and $y_{2}$ are the outputs of the two nonlinear devices. 


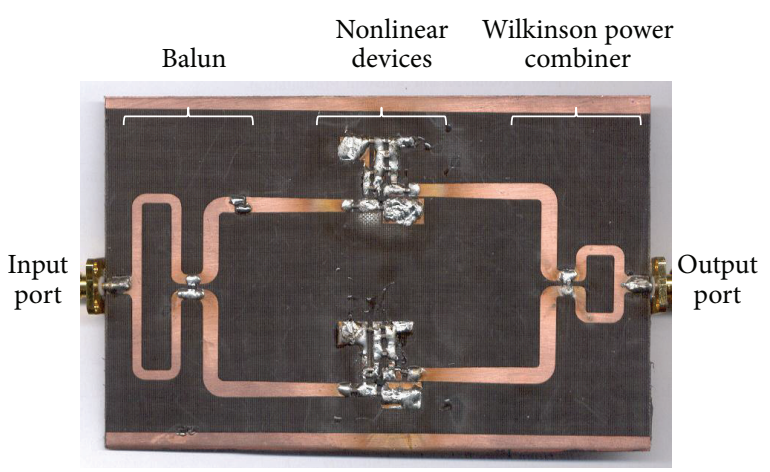

(a)

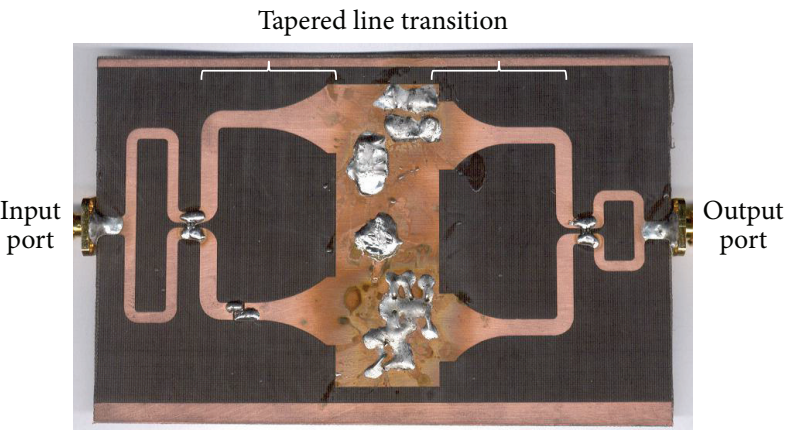

(b)

FIGURE 3: (a) Top view of the proposed frequency doubler. (b) Bottom view of the proposed frequency doubler.

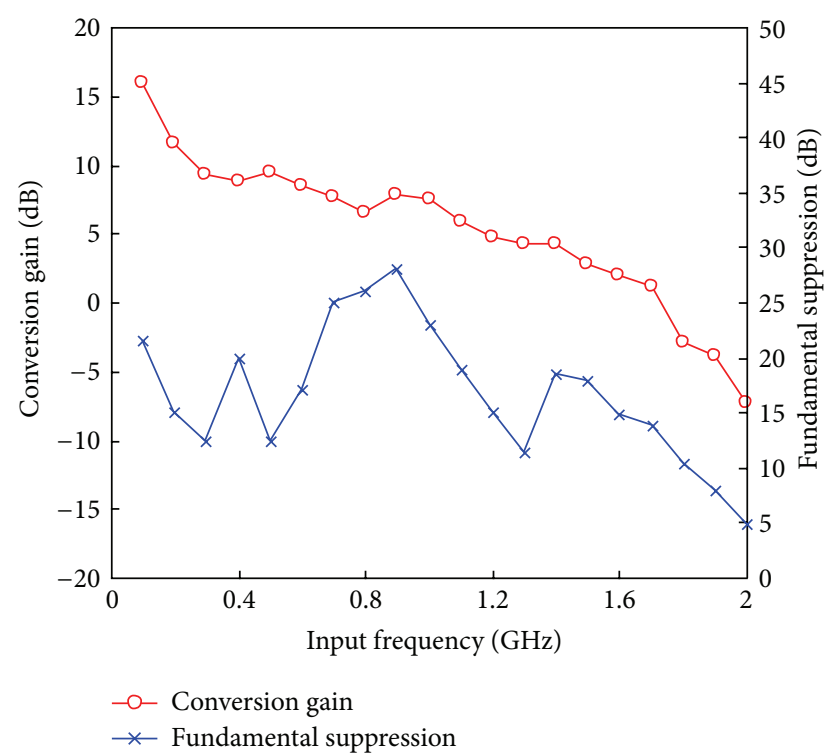

FIGURE 4: Frequency response of the conversion gain and fundamental suppression with fixed input power of $-30 \mathrm{dBm}$ and fixed biasing condition.

The input balun divides the input signal into two equal in magnitude but $180^{\circ}$ out-of-phase signals that are the inputs of the two nonlinear devices; namely, $x_{2}=-x_{1}$. The outputs of two nonlinear devices become

$$
\begin{gathered}
y_{1}=a_{1} x_{1}+a_{2} x_{1}^{2}+a_{3} x_{1}^{3}+a_{4} x_{1}^{4}+a_{5} x_{1}^{5}+\cdots, \\
y_{2}=a_{1}\left(-x_{1}\right)+a_{2}\left(-x_{1}\right)^{2}+a_{3}\left(-x_{1}\right)^{3} \\
+a_{4}\left(-x_{1}\right)^{4}+a_{5}\left(-x_{1}\right)^{5}+\cdots
\end{gathered}
$$

The in-phase Wilkinson power combiner is used to combine the two outputs into one. The eventual output becomes

$$
\begin{gathered}
y=y_{1}+y_{2}, \\
y=a_{2} x_{1}^{2}+a_{4} x_{1}^{4}+a_{6} x_{1}^{6}+\cdots .
\end{gathered}
$$

The fundamental signal and all the odd-order harmonic signals are cancelled in the ideal case. Practically, these signals are not completely suppressed since the balun always introduces magnitude and phase errors.

In this study, a wideband frequency doubler is proposed using the parallel-strip balun. The output Wilkinson power combiner achieves wide impedance bandwidth. The proposed frequency doubler was fabricated on the dielectric substrate with a relative permittivity of 4.6 and substrate thickness of $1.0 \mathrm{~mm}$. Impedance matching networks could be used to enhance the conversion gain at certain narrow range of frequency, since impedance matching network is frequency dependent. The network is the critical factor to limit the bandwidth of the frequency doubler. In this study, no matching networks are designed for the transistors to ensure the frequency doubler working over wide frequency range. Figure 4 shows the frequency response of the conversion gain with fixed input power of $-30 \mathrm{dBm}$ and fixed biasing condition. The significant conversion gain is achieved over input frequency from $0.1 \mathrm{GHz}$ to $1.7 \mathrm{GHz}(178 \%$ relative bandwidth). The input power is fixed at $-3 \mathrm{dBm}$. It achieves $7.4 \mathrm{~dB}$ conversion gain and $23 \mathrm{~dB}$ fundamental suppression at $1.0 \mathrm{GHz}$. Figures 5 and 6 show the measured conversion gains and the measured supply currents against the input fundamental power up to $-3 \mathrm{dBm}$, respectively. Three different input signal frequencies, $0.8 \mathrm{GHz}, 1.0 \mathrm{GHz}$, and $1.2 \mathrm{GHz}$, are chosen, and the output frequencies are $1.6 \mathrm{GHz}, 2.0 \mathrm{GHz}$, and $2.4 \mathrm{GHz}$, respectively.

\section{Conclusion}

The parallel-strip phase inverter with simple and compact impedance matching networks is designed. The parallelstrip balun consisting of a Wilkinson power divider, a phase inverter, and a section of delay line is achieved with wideband differential output. The balanced frequency doubler using the proposed balun was designed and fabricated on a single piece of printed circuit board. A wideband frequency doubler based on the parallel-strip circuit is demonstrated for the first time. The proposed balun can also be integrated to other balanced devices such as microwave mixers, oscillator, and antennas. 


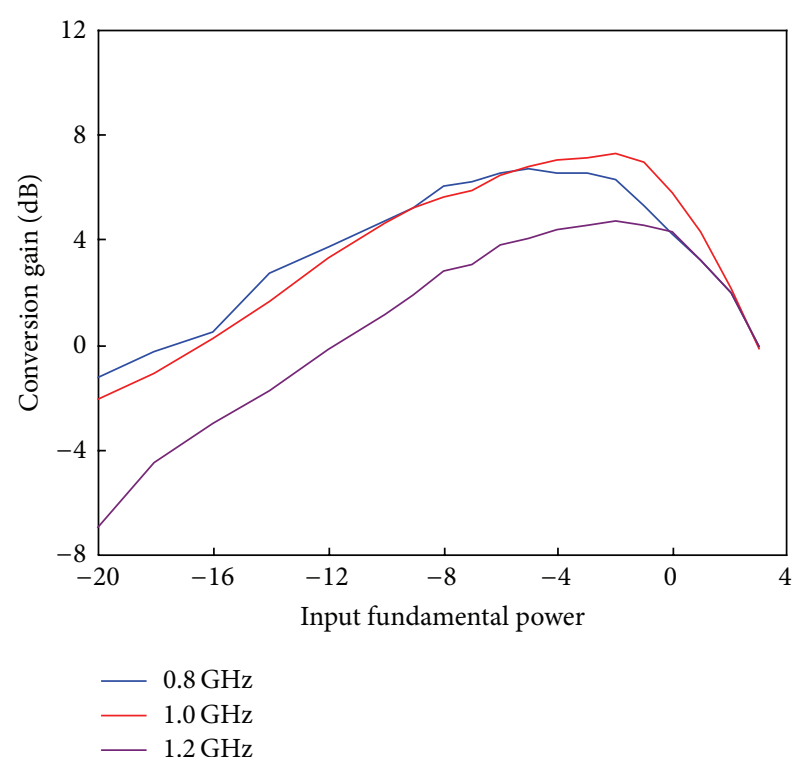

FIGURE 5: Measured conversion gains against input fundamental power with three input frequencies of $0.8 \mathrm{GHz}, 1.0 \mathrm{GHz}$, and $1.2 \mathrm{GHz}$.

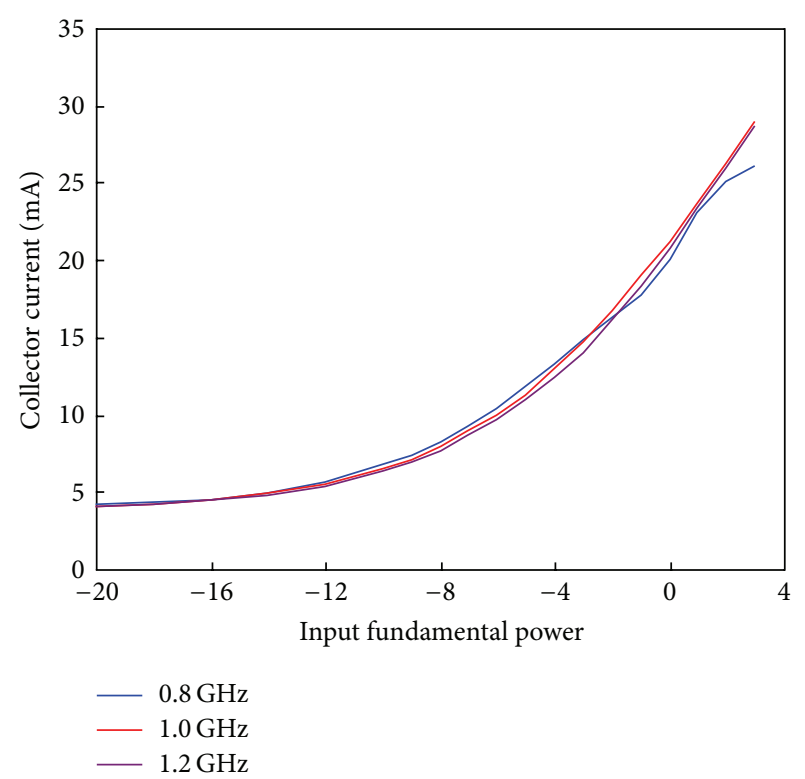

FIGURE 6: Measured current against input fundamental power with three input frequencies of $0.8 \mathrm{GHz}, 1.0 \mathrm{GHz}$, and $1.2 \mathrm{GHz}$.

\section{References}

[1] Z. Y. Zhang, Y. X. Guo, L. C. Ong, and M. Y. W. Chia, "A new wide-band planar balun on a single-layer PCB," IEEE Microwave and Wireless Components Letters, vol. 15, no. 6, pp. 416-418, 2005.

[2] Y. Liu, T. Yang, Z. Yang, and J. Chen, "A 3-50 GHz ultrawideband PHEMT MMIC balanced frequency doubler," IEEE Microwave and Wireless Components Letters, vol. 18, no. 9, pp. 629-631, 2008.
[3] Q. Xue, X. Y. Zhang, and C. H. K. Chin, "A novel differentialfed patch antenna," IEEE Antennas and Wireless Propagation Letters, vol. 5, no. 1, pp. 471-474, 2006.

[4] M. A. Antoniades and G. V. Eleftheriades, "A broadband Wilkinson balun using microstrip metamaterial lines," IEEE Antennas and Wireless Propagation Letters, vol. 4, no. 1, pp. 209212, 2005.

[5] S. G. Kim and K. Chang, "Ultrawide-band transitions and new microwave components using double-sided parallel-strip lines," IEEE Transactions on Microwave Theory and Techniques, vol. 52, no. 9, pp. 2148-2152, 2004.

[6] K. W. Wong, L. Chiu, and Q. Xue, "Wideband parallel-strip bandpass filter using phase inverter," IEEE Microwave and Wireless Components Letters, vol. 18, no. 8, pp. 503-505, 2008.

[7] L. Chiu and Q. Xue, "Wideband parallel-strip $90^{\circ}$ hybrid coupler with swap," Electronics Letters, vol. 44, no. 11, pp. 687-688, 2008.

[8] K. L. Deng and H. Wang, "A miniature broad-band pHEMT MMIC balanced distributed doubler," IEEE Transactions on Microwave Theory and Techniques, vol. 51, no. 4, pp. 1257-1261, 2003.

[9] E. Camargo, Design of FET Frequency Multipliers and Harmonic Oscillators, Artech House, Norwood, Mass, USA, 1998.

[10] Zeland Software, Inc., Std. IE3D. 

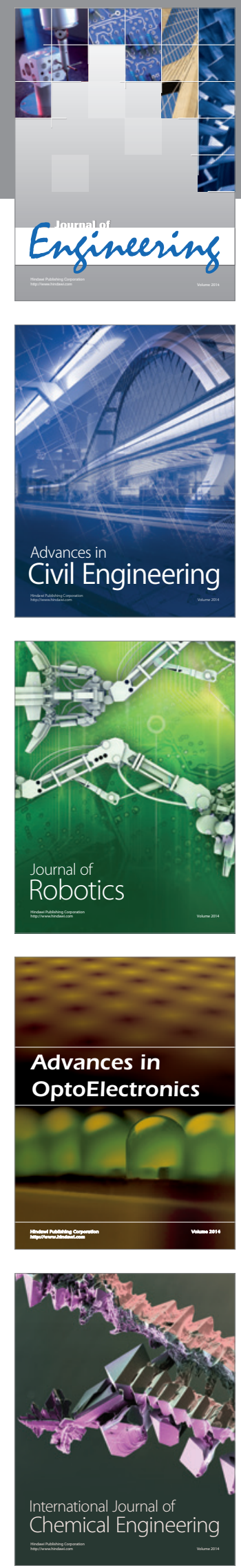

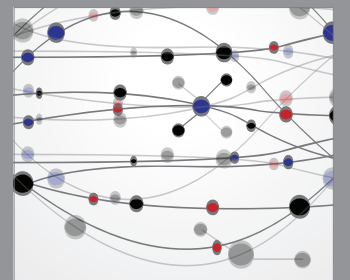

The Scientific World Journal
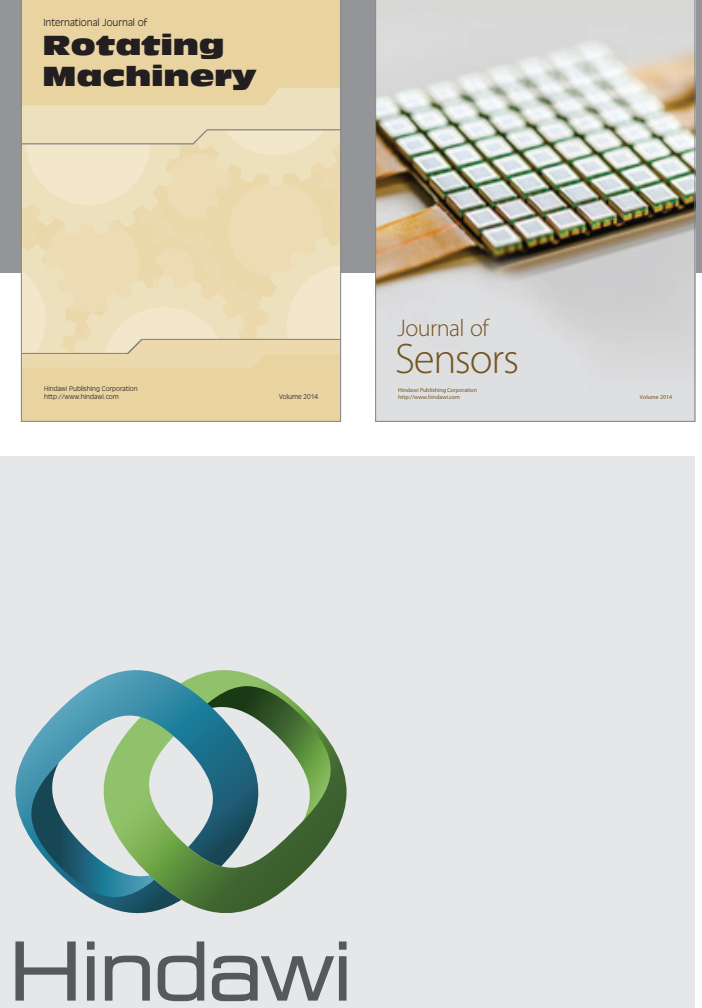

Submit your manuscripts at http://www.hindawi.com
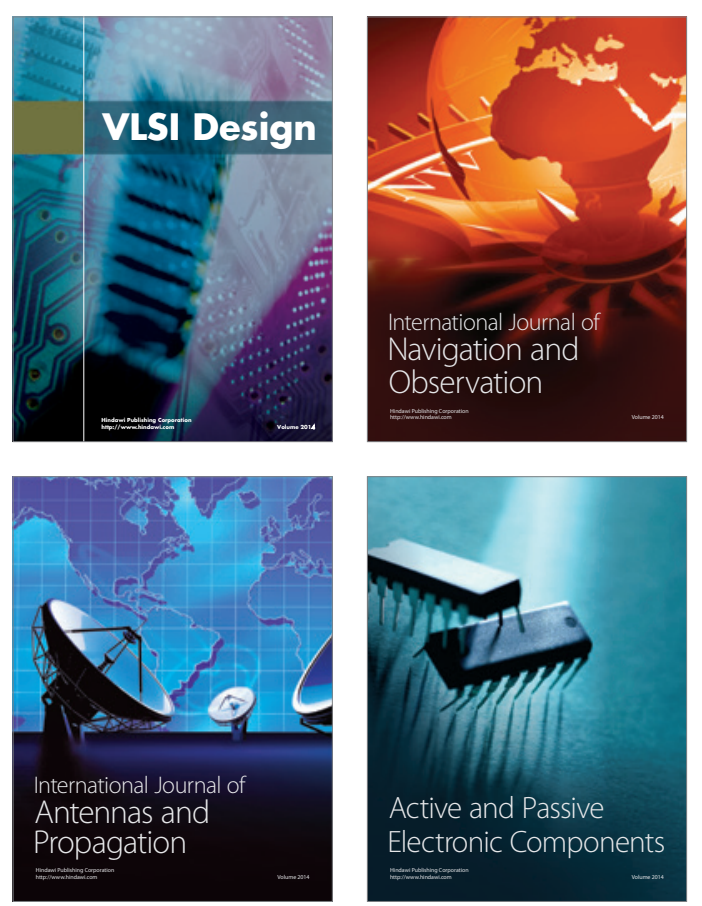
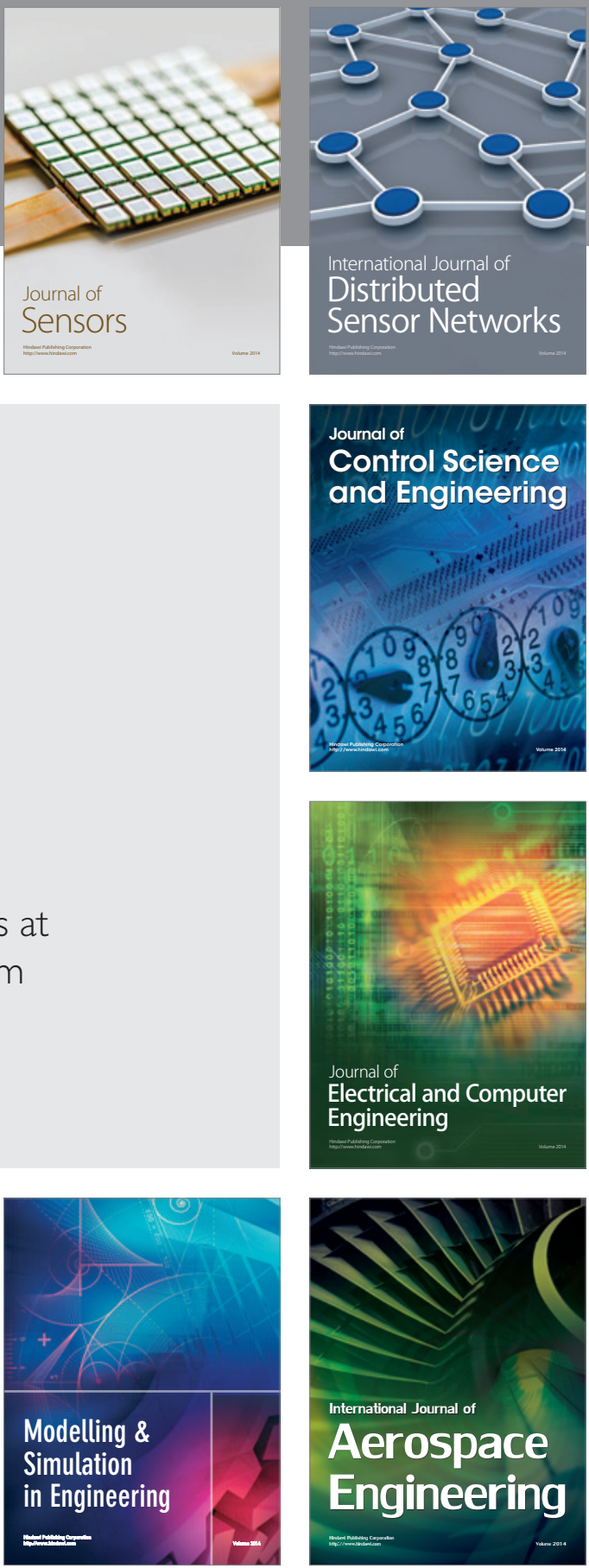

Journal of

Control Science

and Engineering
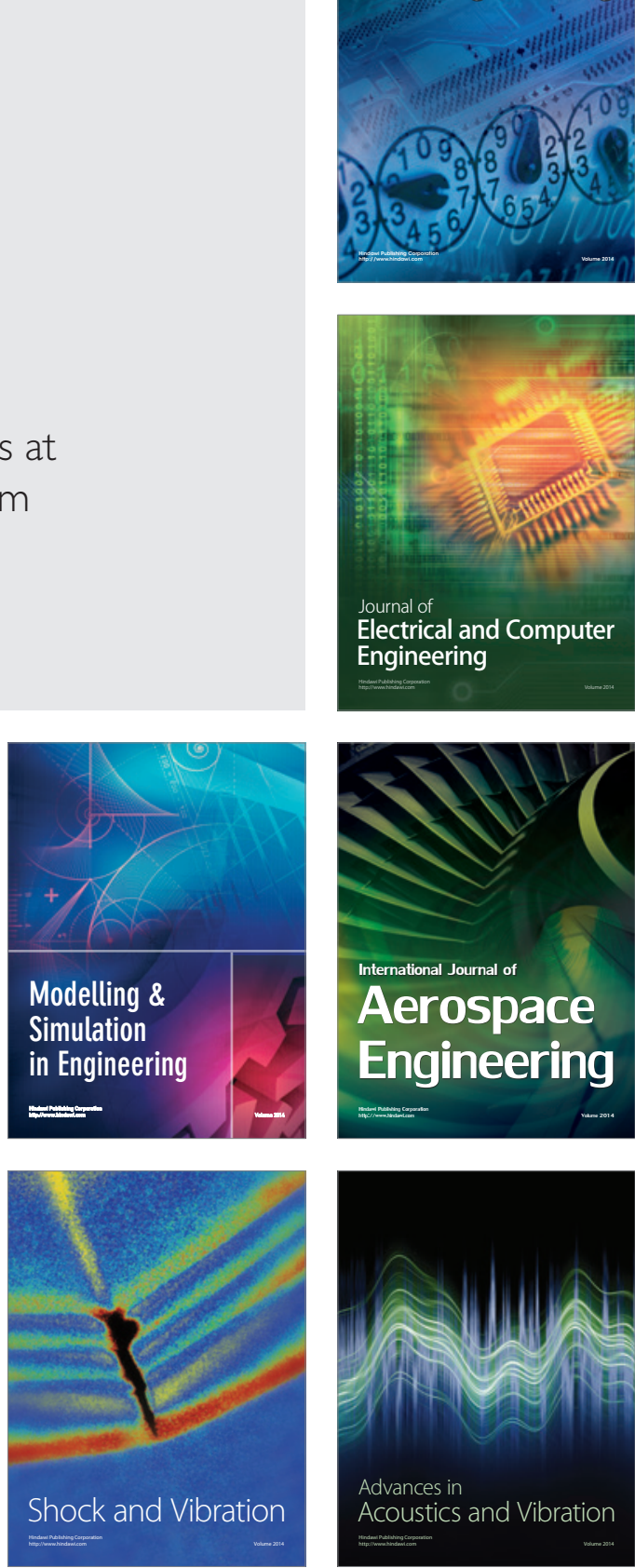